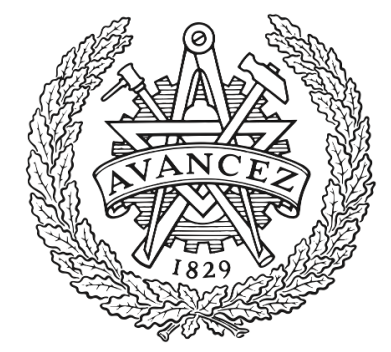

CHALMERS

UNIVERSITY OF TECHNOLOGY

\title{
Enabling Technologies for Optical Data Center Networks: Spatial Division Multiplexing
}

Downloaded from: https://research.chalmers.se, 2023-04-26 12:24 UTC

Citation for the original published paper (version of record):

Zhang, L., Chen, J., Agrell, E. et al (2020). Enabling Technologies for Optical Data Center Networks: Spatial Division Multiplexing. Journal of Lightwave Technology, 38(1): 18-30. http://dx.doi.org/10.1109/JLT.2019.2941765

N.B. When citing this work, cite the original published paper. 


\title{
Enabling Technologies for Optical Data Center Networks: Spatial Division Multiplexing
}

\author{
Lu Zhang, Member, IEEE, Jiajia Chen, Senior Member, IEEE, Erik Agrell, Fellow, IEEE, Rui Lin, and \\ Lena Wosinska, Senior Member, IEEE
}

(Invited Tutorial)

\begin{abstract}
With the continuously growing popularity of cloud services, the traffic volume inside the data centers is dramatically increasing. As a result, a scalable and efficient infrastructure for data center networks (DCNs) is required. The current optical DCNs using either individual fibers or fiber ribbons are costly, bulky, hard to manage, and not scalable. Spatial division multiplexing (SDM) based on multicore or multimode (few-mode) fibers is recognized as a promising technology to increase the spatial efficiency for optical DCNs, which opens a new way towards high capacity and scalability. This tutorial provides an overview of the components, transmission options, and interconnect architectures for SDM-based DCNs, as well as potential technical challenges and future directions. It also covers the co-existence of SDM and other multiplexing techniques, such as wavelength-division multiplexing and flexible spectrum multiplexing, in optical DCNs.
\end{abstract}

Index Terms-Fiber-optical communication, multicore fiber, few-mode fiber, multiplexing and demultiplexing, network architecture, optical data center networks, resource allocation, spatial division multiplexing, switching.

\section{INTRODUCTION}

$\mathrm{D}$ ATA centers (DCs) are playing key roles in Internet service delivery for an ever-increasing number of customers and devices [1-3], which raise strict requirements on interconnection networks for DCs in terms of capacity, power consumption and latency. Regarding the capacity requirement, DC traffic will continue to dominate the Internet traffic in the foreseen future, and a clear trend of such traffic is transforming

Manuscript received xx xx, 2019, revised xx xx, 2019, accepted xx xx, 2019; This work was partly supported by Swedish Research Council (VR), the Swedish Foundation for Strategic Research (SSF), Göran Gustafsson Foundation, the Celtic-Plus sub-project SENDATE-EXTEND \& SENDATE FICUS, H2020 5GPPP 5G-PHOS research program (ref.761989), European Commission through the FP7 project MIRAGE (ref.318228), SJTU State Key Laboratory of Advanced Optical Communication System and Networks Open project 2018GZKF03001. (corresponding author: Lena Wosinska)

L. Zhang is with the College of Information Science and EE, Zhejiang University, Hangzhou 310027, China; and also with State Key Laboratory of Advanced Optical Communication System and Networks, Shanghai Jiao Tong University, Shanghai 200240, China. (e-mail: zhanglu1993@zju.edu.cn; luzhang_sjtu@sjtu.edu.cn).

J. Chen, E. Agrell, R. Lin, and L. Wosinska are with the Department of Electrical Engineering, Chalmers University of Technology, Gothenburg, Sweden. (e-mails: agrell@chalmers.se, ruilin@chalmers.se; jiajiac@ chalmers.se; wosinska@ chalmers.se). from the conventional 'north-south' direction that sends/receives data to/from outside of the DCs to the 'east-west' direction corresponding to the traffic that stays locally inside the DC and is predicted to represent $85 \%$ of total DC traffic by 2021 [3-4]. Thus, providing high capacity to the intra-DC network is of great importance. Hereafter, we refer to the DC network (DCN) as the one that handles the traffic within a DC. Moreover, the DCs are predicted to consume around $3 \sim 13 \%$ of global electricity in 2030 [5]. Communications are essential in DCs, and therefore there is a strong need for power efficient techniques in DCNs. To be able to support future generations of communication use cases and scenarios, which increasingly involve cloud facilities [6-7], low latency is required for end-to-end connections. The extreme examples are autonomous driving and remote surgery, where ultra-low latency is needed.

To address the requirements on capacity, power consumption and latency, optical communication techniques have been introduced in DCNs [8]. In addition, optical multiplexing techniques, such as spatial division multiplexing (SDM) and wavelength division multiplexing (WDM), have been considered to increase the transmission capacity in DCNs.

SDM uses the controllable arrangement of optical signals in the spatial domain. It has a great potential for DCNs because it offers ultra-high capacity and good compatibility with WDM techniques. The initial stage of deploying SDM in DCNs was to deploy parallel optical fibers connecting servers and/or racks, and then the optical fiber bundles/ribbons were introduced to reduce the cabling complexity [8]. To date, the parallel optical fibers and fiber bundles/ribbons are commonly deployed in commercial DCs. Moreover, O-band coarse WDM techniques, e.g., $8 \times 50 \mathrm{Gbps}$ for 400 Gigabit Ethernet (GbE) interface [9], are commercially used in combination with the aforementioned SDM techniques for DCN interconnections to further increase the capacity [10]. However, DCNs are continuously coping with ever-increasing capacity demand, and the service providers are looking for higher transmission speed in DCNs, e.g. 1TbE and beyond [11]. Thus, a next step for increasing the DCN capacity still needs to be investigated.

Recently, SDM techniques using multicore fiber (MCF) or few-mode fiber (FMF) have gained a lot of attention. By arranging numerous spatial channels in a single fiber, the spatial efficiency, defined as the throughput per unit of 
cross-section area, can be greatly improved and more spatial channels can be transmitted in parallel to increase the capacity. Moreover, the channels using coarse or dense WDM techniques [12] can be transmitted in the MCF/FMF to improve the spectral efficiency when needed [13]. In [14], the authors benchmarked common DCN topologies [15] under SDM and WDM techniques in terms of network throughput, resource utilization, blocking probability, cost and power consumption. It has been shown that SDM and WDM techniques exhibit comparable performance for different topologies, and it is feasible to combine SDM and WDM techniques in DCNs.

This tutorial paper concentrates on the SDM as an enabling technology for high-performance DCNs. We outline various SDM components, transmission options and interconnect architectures, and highlight their development trend. We also present technical insights into the co-existence of SDM with other multiplexing techniques, such as WDM and flexible spectrum multiplexing, in optical DCNs.

The rest of this paper is organized as follows. Section II considers the device level and presents a review of key SDM components for DCNs, including SDM fibers, multiplexers/demultiplexers, and switches. Section III describes the state-of-the-art SDM transmission options for DCNs that have already been experimentally demonstrated. The design of SDM transceivers, the modulation formats and signal processing algorithms in SDM enabled DCN links are also discussed. In Section IV, the network aspects for SDM based DCNs are presented, including interconnect architectures along with the resource allocation strategies, where the potential technical challenges and future research directions are also discussed. Finally, Section V summarizes the paper and provides the final conclusions.

\section{KEY SDM COMPONENTS FOR DCNS}

This section outlines the key SDM components for DCNs. DCNs have a relatively short reach (typically up to a few kilometers) and hence no strong needs for amplification [16]. Therefore, SDM amplifiers are not considered in this section. The other relevant components, i.e., SDM fibers, SDM multiplexers (MUX) and demultiplexers (DEMUX), and SDM switches are described in the following.

\section{A. SDM Fibers}

Current DCNs use either individual parallel optical fibers or fiber bundles/ribbons [17], due to their better energy and spatial efficiency than electrical cables. Parallel optical fibers and fiber bundles/ribbons, which use a bundle of conventional single-mode fibers (SMFs) packed together, can be considered as a straightforward way to realize SDM. However, they are still not spatially efficient enough, and the photonics integration of them is difficult. Instead, MCF and FMF can improve the spatial efficiency, whereas higher component cost and more complex installation may arise in such DCNs, since the technologies are presently less mature. In the following, we mainly focus on MCF and FMF types of SDM.

Multicore fiber $(M C F)$ can be mainly categorized as uncoupled, weakly-coupled, or strongly-coupled MCFs [18-20].
A widely considered MCF type is the 7-core hexagonal arrangement shown in Fig. 1 [21], where a marker is used for core identification. In the design of MCFs, a trench-assisted structure is proposed for reducing the coupling among the cores. By surrounding each core with a low-index trench layer, the electric field distribution in each core is suppressed and the overlap of electric field among adjacent cores becomes small [22-23]. As a result, the inter-core crosstalk (IC-XT) could be significantly reduced in the trench-assisted MCF. For example, the IC-XT of the commercially available 7-core fiber with a trench-assisted structure in [24] is as low as $-45 \mathrm{~dB} / 100 \mathrm{~km}$, which means that the cores of MCFs can be treated almost independently within the typical reach in DCNs.

The advances in MCF fabrication enables higher spatial efficiency with a novel cross-section geometry design, such as two-pitch 10-core fiber [25], dual-ring 12-core fiber [26], and hexagonal 30-core fiber [27]. Linearly arrayed MCFs with core arrangements in a rectangular shape [28, 39], as shown in Fig. 2, are well suited for integration with silicon photonic transceivers. For instance, a 100-Gbps parallel single-mode silicon photonic system uses surface coupling with an 8-core MCF, using 4 cores for transmission and the other 4 for reception [28].

Uncoupled or weakly-coupled MCFs having a low core count shows a relatively low IC-XT [37]. Increasing the core count in MCFs, keeping the cladding diameter fixed, offers a higher spatial efficiency. For long-term reliability, low cabling cost, and compatibility with the current SMF fabrication process [18], it is advantageous to keep the standard $125 \mu \mathrm{m}$ cladding diameter (ITU-T G.657 A1 [29]). However, when the core count increases for a given cladding diameter, the coupling between adjacent cores gets stronger, often referred to as strongly-coupled MCFs. Strongly-coupled MCFs typically show high coupling between adjacent cores, inducing high IC-XT and therefore deteriorating the signal transmission performance. The peak capacity is attained at a core count of

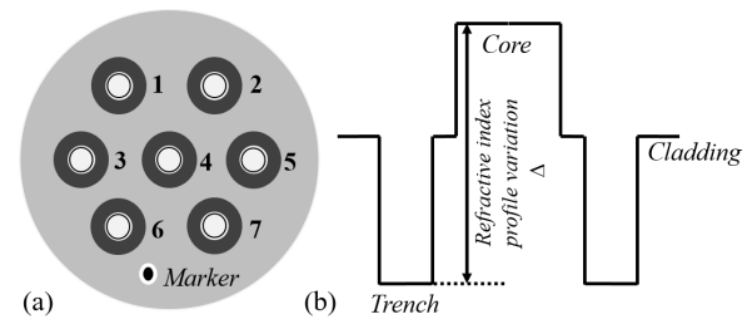

Fig. 1. (a) The cross-section view of the 7-core MCF and (b) the refractive index profile of MCF.
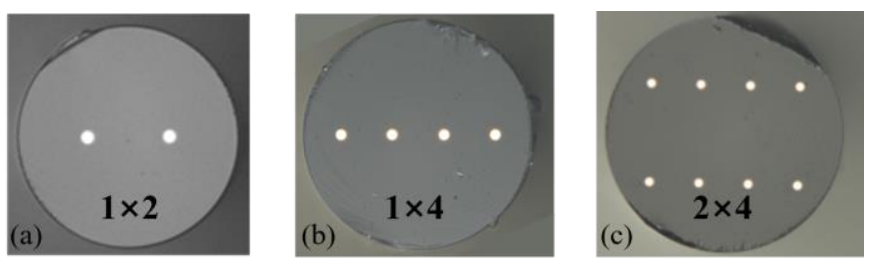

Fig. 2. (a) The cross-section view of the MCF with linear arrayed cores in the arrangements of (a) $1 \times 2$, (b) $1 \times 4$, (c) $2 \times 4$ [39]. (C) IEEE, reprinted with permission.) 
about 25 30 depending on the signal-to-noise ratio (SNR) [30]. To some extent, the IC-XT can be overcome by digital signal processing (DSP) techniques, which is discussed in Section III.

The long-term IC-XT is often modelled as independent of the modulation format [30]. The short-term IC-XT fluctuations depend on many factors, including modulation format [30], symbol rate [31], skew between cores [31], operating wavelength [32], temperature [33], and pseudo random binary sequence (PRBS) length [33]. Carrier-supported signals, such as intensity modulated signals, induce the IC-XT that varies with time [31]. The distortion of the transmitted signals is caused either by transmission through large inter-core skew MCFs or by using high symbol rates [31,34]. It may be also the reason of the IC-XT fluctuations. Besides, longer PRBS and lower temperature contribute to a lower IC-XT [33]. The IC-XT dynamics need to be considered in the deployment of MCFs. Furthermore, the tolerance to crosstalk depends strongly on the modulation format, as constellation points using higher-order modulation lie closer together and are more easily confused in the receiver [35-36].

In the first generation of optical DCNs, multimode fiber $(M M F)$ was used to carry the optical signals. Although such fibers support tens of linear polarization modes [16], this degree of freedom was originally not exploited. The transmission quality in MMF is influenced by mode dispersion, inter-mode crosstalk, and interference induced by differential mode delay. As a result, the allowed transmission distance is limited to a few hundred meters. To mitigate these impairment, and simultaneously increase spatial efficiency, MMFs have been recently considered in an SDM context, transmitting independent data on different modes. In such systems, multimode multiplexers (MUXs) and demultiplexers (DEMUXs) are applied for signal recovery, along with multiple-input multiple-output (MIMO) DSP. As a simpler alternative, few-mode fiber $(F M F)$ utilizes a small number of linear polarization modes as spatial channels, by optimizing the design of the refractive index of the fiber core and cladding. Although FMFs [38-39] can reduce the DSP complexity compared with MMFs, the MIMO-DSP is also required to process the inter-mode crosstalk. Few-mode multicore fiber $(F M-M C F)$ multiplexing several modes in each core is a combination of MCF and FMF, which increases the spatial efficiency compared with using either of them. Compared to MMF, the complexity of DSP can be significantly reduced in FM-MCFs thanks to the simplified MIMO DSP matrix [16], while the same number of the spatial channels can be achieved. On the other hand, the transceivers, switches and MUX/DEMUX modules tailored for the FM-MCFs are still under development.

There are also several novel SDM fiber techniques, which can potentially be appropriate for the future DCNs. Firstly, to overcome the mode coupling problem, an elliptical-core FMF has been proposed for SDM transmission [40]. The propagation constants for the linear polarization modes in each mode group are different due to the asymmetrical shape of the core, and the mode coupling can be suppressed. Secondly, so-called hollow-core photonic band gap fibers have been designed
[41-42], which guide the light based on the photonic band-gap mechanism through a carefully designed mesh of tiny air- or vacuum-filled tubes. This type of fibers enables higher speed of light and supports transmission carried by $2 \mu \mathrm{m}$ wavelength, but it suffers from higher power loss compared to the conventional fibers. Moreover, extremely high mechanical precision in manufacturing is required.

\section{B. SDM MUX/DEMUX Modules}

The components that connect a number of single-mode single-core fibers to the corresponding spatial channels of SDM fibers are referred to as SDM MUX/DEMUX modules. They are important for SDM deployment in DCNs, since they can make SDM compatible with existing systems, reduce the capital expenditures, and improve the scalability and flexibility when upgrading the DCNs. Besides, SDM MUX/DEMUX modules can bring fine switching granularity in DCNs, allowing for switching on a per-spatial-channel basis.

We will discuss SDM MUX/DEMUX modules for MCF, FMF, and MMF separately. First, for MCFs, the SDM MUX/DEMUX modules (also known as fan-in/fan-out modules) connect a number of single-mode single-core fibers to different cores in an MCF. As shown in Fig. 3, the operation principle of SDM MUX/DEMUX modules connecting MCF can be mainly divided into free-space optics [43], fused taper [44], fiber bundle [45], and compact waveguide coupling [46].

Using free-space optics (see Fig. 3a), a single lens is applied to couple the single-core fiber's outputs to the corresponding $\mathrm{MCF}$ cores. It is realized by putting the end facet of the MCF at the front focal point of the single lens. The fabricated module with $40 \mathrm{~mm}$ (diameter) and $62 \mathrm{~mm}$ (length) presented in [43] exhibits IC-XT below -50dB. The insertion loss for each port is lower than $0.6 \mathrm{~dB}$ and the difference in coupling loss is below $0.4 \mathrm{~dB}$. Compared with direct fiber-to-fiber coupling, this design is tolerant to a shift in the core position. However, this scheme is bulky and requires sophisticated opto-mechanical operations.

The fused-taper scheme (see Fig. 3b) utilizes the elongation process to consolidate the single-core fibers with the corresponding MCF. The diameter of the module presented in [44] is $0.72 \mathrm{~mm}$ and its length is $35 \mathrm{~mm}$. The maximum insertion loss of this module is $4.7 \mathrm{~dB}$ and the worst IC-XT is $-45 \mathrm{~dB}$ at $1550 \mathrm{~nm}$. The fiber-bundle-based fan-in/fan-out fabrication scheme (see Fig. 3c) uses chemical etching of the single-core fibers until the cladding diameter matches the corresponding core pitch of the MCF. The device reported in [45] is 5mm wide and $32 \mathrm{~mm}$ long. It is characterized by IC-XT lower than $-50 \mathrm{~dB}$ and by the insertion loss around $0.6 \mathrm{~dB}$. These two kinds of schemes are quite compact and its fabrication process is cost-efficient. The crosstalk needs to be carefully controlled during the fabrication.

The compact waveguide coupling scheme realizes fan-in/fan-out functionalities by inscribing spatially isolated waveguides that connect each core of the MCF to a particular SMF. Fig. 3d illustrates such a fan-out module using a laminated polymer waveguide [46]. The insertion loss of the 19 cores in the device in [46] varies from $0.2 \mathrm{~dB}$ to $1.8 \mathrm{~dB}$ and the 
IC-XT of all the 19 cores is lower than -40dB. Compared with the aforementioned schemes shown in Figs. 3a-c, the waveguide coupling scheme exhibits merits in terms of compactness and simple large-scale fabrication. The MCF with a compact fan-in/fan-out module, which couples an MCF to laser diodes (LDs) and photodiodes (PDs) with pluggable connections, can also be a feasible solution for DCN applications. All the mentioned module types have been commercialized by companies like OptoQuest [47], Optoscribe [48], and Chiral Photonics [49]. The crosstalk, insertion loss, and reflection loss have been optimized.

Here we present SDM MUX/DEMUX modules for FMF and MMF together, both of which are for mode MUX/DEMUX. Such modules connect single-mode single-core fibers to individual transmission modes in a fiber. The operation principles of the MUX/DEMUX modules can be mainly divided into mode conversion [50-52], index matching [53-54], and photonic lantern schemes [55].

In the mode-conversion scheme, the single mode in the conventional fibers is converted to linear polarization modes by phase plates or long period gratings [50], after which the modes are combined again using beam combiners. The mechanism of this scheme is simple, but it suffers from high power loss induced by conversion and combination. The simultaneous mode-conversion schemes convert a single mode into multiple modes by free-space optics and a reflective phase plate [51] or grating couplers [52]. The grating coupler scheme requires

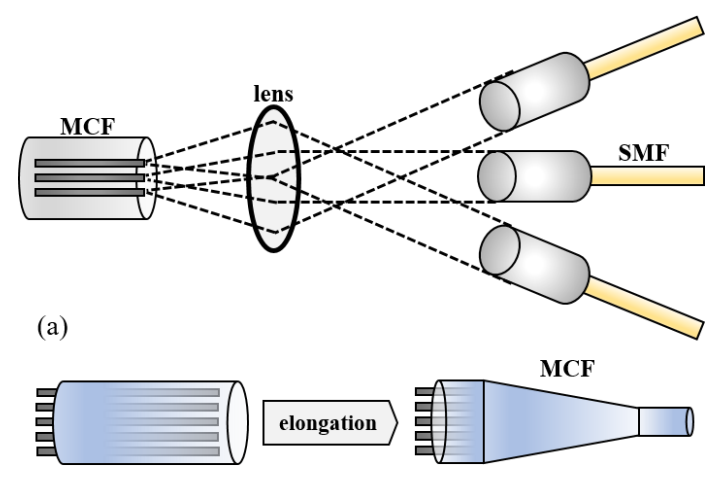

(b)

(c)

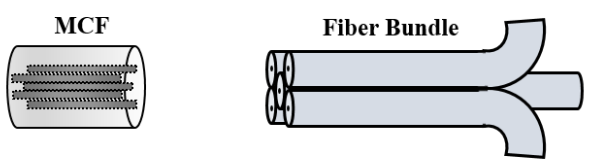

(d)

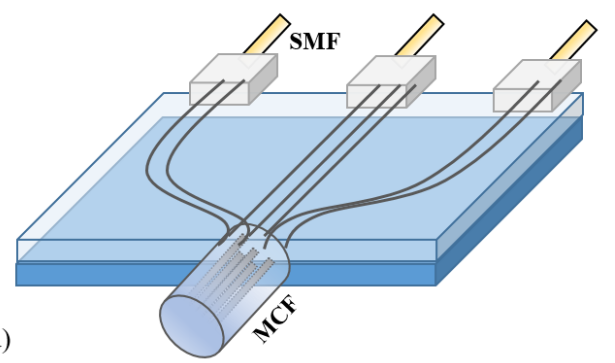

Fig. 3. The SDM fan-in/fan-out fabrication process with (a) free space optics scheme, (b) fused taper scheme, (c) fiber bundle scheme, and (d) compact waveguide coupling scheme. control of phase tuners and input light polarization.

In the index-matching scheme, asymmetric directional couplers based on fused fibers [53] or other waveguides [54] are used to couple multiple modes into a common multimode port. The conversion efficiency is high, but the fabrication process is more complex than for the mode-conversion scheme.

Finally, the photonic-lantern scheme [55] is realized with a mechanism similar to the aforementioned fused-taper scheme, where the cores are allocated closely enough for strong core-coupling [55].

\section{SDM Switches}

To meet the demands for flexible service provisioning in DCNs, switching and add/drop multiplexing in the spatial domain need to be provided. SDM switching can be considered in the wavelength and/or time domain in order to enable compatibility with the existing infrastructure. There are various approaches to realize switching functionality in the spectrum and space domains. The straightforward way is to use the aforementioned SDM MUX/DEMUX modules connecting MCF/FMF/MMF at the input/output ports of the traditional switching modules [56]. Inside the switching node, the input signals are first separated into independent spatial channels, and then the micro-electro-mechanical system (MEMS) mirrors or liquid crystal on silicon (LCoS) [57-59] based wavelength/spectrum selective switches (WSS/SSS) and/or optical cross-connects are used to switch the demultiplexed spatial channels.

An experimental demonstration of joint-spectral-spatial switching using an LCoS-based WSS was presented in [58]. A heterogeneous SDM network was considered, applying 3 types of SDM fiber spans and a WSS with a large port count supporting 6 modes. A WSS integrated with FMFs was experimentally demonstrated in [59]. By arranging the WSS input/output fibers in an array, a set of inputs to an LCoS-based WSS can be steered onto different sets of outputs. Combining the input/output SMF groups using SDM MUX/DEMUX allows switching all the spatial channels to their destinations. This technique reformats the channels of the SDM fiber without sacrificing the hardware complexity of the WSS. Moreover, by arranging the spatial channels in a $2 \mathrm{D}$ array, the switching

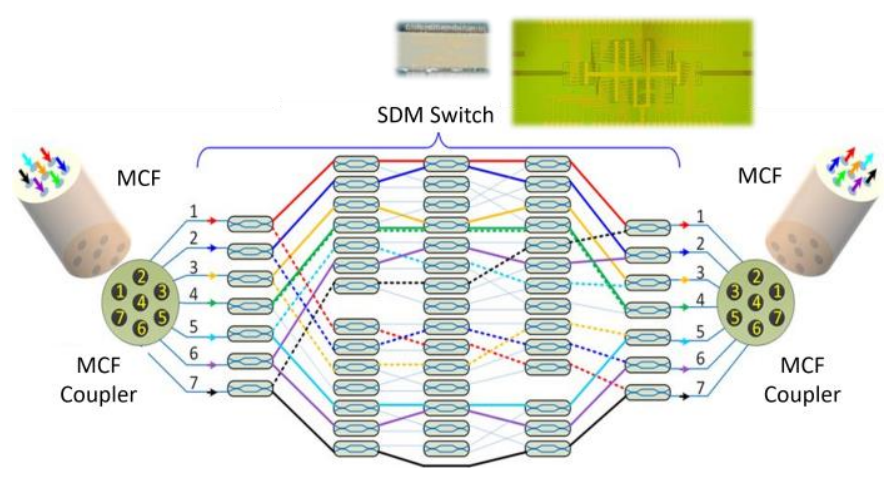

Fig. 4. A silicon photonic integrated circuit for an SDM switch with MCF [61]. (C) Springer Nature, reuse permitted by the CC BY license.) 
action can be extended to any rows of fibers of the array [58]. This free-space switching scheme requires precise opto-mechanical operations to realize fine spectral-spatial switching granularity and high resolution that can greatly reduce the IC-XT and the spectral line spacing.

There are also some works on SDM switches for MCFs. In [60], directional bending of a long-period grating in MCFs to realize reconfigurable inter-core broadcasting and switching is presented, which again requires precise opto-mechanical operations. Fig. 4 shows an integrated SDM switch module using a silicon-photonic integrated circuit [61]. It is fabricated on a silicon-on-insulator platform. The demonstrated silicon photonic integrated circuit is composed of a $7 \times 7$ switch and MCF couplers with a low-loss grating coupler array. An insertion loss of $4.5 \mathrm{~dB}$ at $1546 \mathrm{~nm}$ is experimentally demonstrated [61], which includes the coupling loss of the input and output MCF couplers, the waveguide propagation loss, and all losses caused by the Mach-Zehnder interferometers and cross intersections. The proposed design can be upgraded to a reconfigurable optical add/drop multiplexer capable of switching among several MCFs. A beam steering MCF based SDM switch is proposed and analyzed in [62-63]. In the basic layout of the switch, fiber-pigtailed collimators arranged in regular 2D arrays are individually steered by voltage-controlled actuators [64]. The SDM switch function is achieved by using a single-sided 2D array of mirror and lens to direct beams between any pair of input and output ports of the MCF collimators.

\section{TRANSMisSion Aspects: PRINCIPLES AND Options}

This section addresses a point-to-point link composed of the key SDM components described in Section II. Different aspects that impact SDM transmission are reviewed, including SDM transceivers, modulation formats, and the corresponding DSP algorithms along with the system configurations.

\section{A. SDM Transceivers}

In long-haul transmissions [65], heterodyne or homodyne coherent detection scheme with DSP is employed since cost is often not a very serious concern in core networks. Nevertheless, DCNs favor direct detection scheme now thanks to its low system cost, ease of system configuration, and low DSP complexity. Pluggable transceivers (TRx) are widely used in optical interconnects, which in most cases is a key part of the point-to-point link setup. Currently, TRxs for short reach applications are mainly used together with MMF [18], without employing any SDM. The vertical-cavity surface-emitting laser (VCSEL) offers low power consumption, low fabrication cost, and ease of integration into LD arrays. Multimode VCSELs exciting several modes and emitting the signals on short wavelength $(850 \mathrm{~nm})$ is one option that adopts SDM transmission in DCNs [18]. The MMFs with VCSELs and PDs at short wavelength have been gradually commercialized by industry, such as Broadcom [66] and Santec [67]. However, the transmission distance and capacity of the aforementioned SDM transceivers are limited by the mode dispersion. To the best of our knowledge, the state-of-the-art transmission distance beyond 100Gbps per lane is $550 \mathrm{~m}$ [68] to achieve error-free transmission with forward error correction coding.

To further increase the capacity and transmission reach in SDM based DCNs, MCF/FMF can be an option. However, in FMF the mode dispersion would limit the SDM transmission capability. Instead, the long wavelength SDM transmission which here refers to the $\mathrm{C}$ band around $1.5 \mu \mathrm{m}$, combined with MCF can be considered. At long wavelength, the transceiver design is more mature. The integration of a PD array at long wavelength is easy in fabrication process, and the technical challenge falls on the transmitter side. Regarding the transmitters, the electro-absorption modulated distributed feedback laser (EML), direct modulated laser (DML), and Mach-Zehnder modulator (MZM) have been widely investigated for DCN applications. An integrated transmitter array is a straightforward way to save cost, power consumption and increase scalability. In our previous work [24], we have reported high-speed multicore transmission with long-wavelength $(1.5 \mu \mathrm{m})$ and single-mode VCSEL for DCNs. The properties of the VCSEL are shown in Fig. 5 [24, 69]. It proves that the existing fabrication process is able to offer large modulation bandwidth (e.g., $22 \mathrm{GHz}$ in Fig. 5c) for a single-mode VCSEL working at long wavelength [24, 69-73]. Thus, single-mode MCF with VCSELs and PDs at long wavelength has a potential for large-scale DCNs, where a several kilometers long link is required.

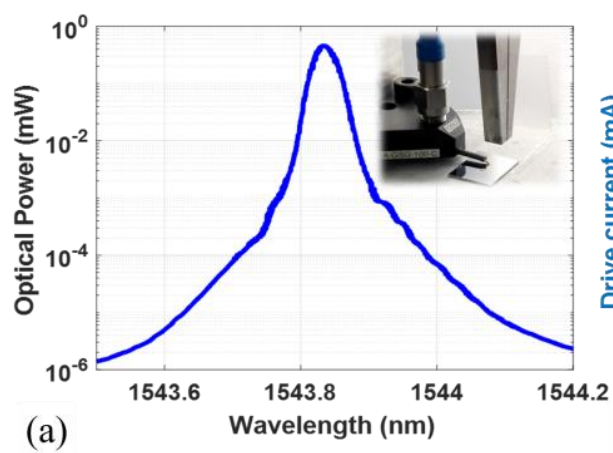

Fig. 5. (a) The optical spectrum, (b) P-I-V curves, and (c) small-signal $\mathrm{S}_{21}$ responses of $1.5 \mu \mathrm{m}$ single mode VCSEL [24]. (@ IEEE, reprinted with permission.)
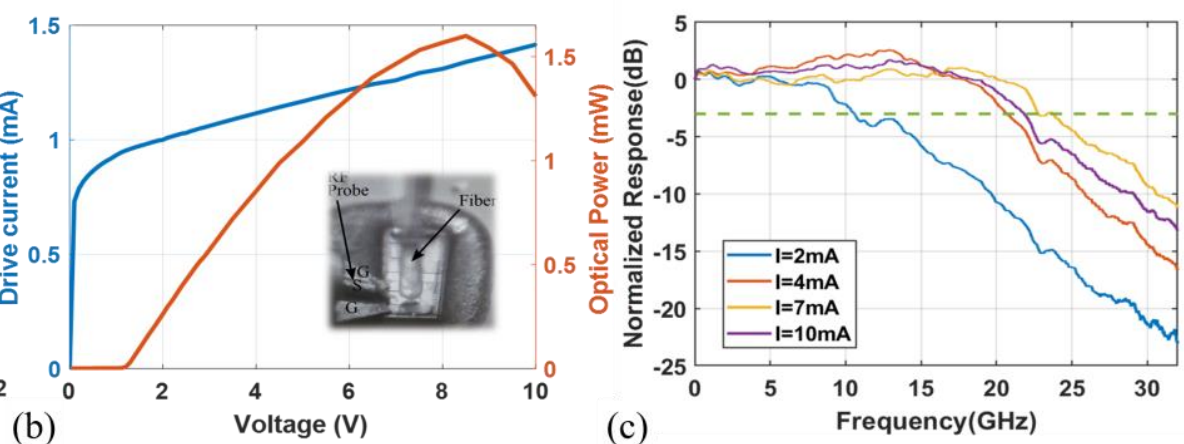

(c) 


\section{B. Modulation Formats and DSP}

Modulation formats, such as non-return to zero (NRZ), electrical duo-binary (EDB), pulse amplitude modulation (PAM), discrete multitone (DMT) have been widely analyzed in DCN interconnects [74]. Although various results on multimode short wavelength DCN interconnects with the aforementioned modulation formats have been studied, simple modulation format like NRZ is favored [18] by industry because it is easy to implement. However, when the capacity requirement and DCN reach is further increased, modulation formats issues need to be re-examined.

Extensive work has been reported on SDM transmission carrying different modulation formats on DCN links, including NRZ [75-77], EDB [75-77], PAM4 [78-81] and DMT [24, 82]. In [77], a BiCMOS chip-based real-time $100 \mathrm{Gbps} / \lambda$ /core NRZ and EDB over 10km 7-core MCF SDM interconnects is experimentally demonstrated. The integrated low complexity transceiver circuits and simple signal processing approach make such a system cost-efficient for the high-speed DCN interconnects. Both NRZ and EDB need 6-tap feed forward equalization (FFE) since they are simple modulation formats and not so sensitive to the inter-symbol-interference (ISI) from the dispersion or inter-mode, inter-core crosstalk. However, the main disadvantage is the large modulation bandwidth required at the transceivers side.

The packaged 100GHz EML based $149 \mathrm{Gbps} / \lambda /$ core PAM4 over $1 \mathrm{~km}$ 7-core MCF SDM link is reported in [78]. Compared with NRZ and EDB, PAM4 provides higher spectral efficiency at the expense of stronger requirements on system SNR. Besides, the EML is difficult to be integrated into transmitter arrays. In [79, 81], using the VCSEL shown in Fig. 5, $100 \mathrm{Gbps} / \lambda /$ core PAM4 transmission is successfully demonstrated over $1 \mathrm{~km}$ and $10 \mathrm{~km}$ 7-core MCF SDM links. Light pre-equalization and post-equalization DSP are required. However, the VCSEL always shows strong nonlinear effects, like chirp combined with dispersion, which makes the system response not flat [24]. As a result, the non-flat response limits the useful bandwidth of single-carrier modulation format. Although the pre-equalization DSP can make the response flat, it is at the expense of reducing the signal SNR in the high channel gain region (always at low frequency).

Total net rates of $726.6 \mathrm{Gbps}$ DMT transmission over $2.5 \mathrm{~km}$ dispersion uncompensated 7-core MCF and 533.1Gbps DMT transmission over $10 \mathrm{~km}$ dispersion compensated 7 -core MCF are experimentally demonstrated in [24, 82]. Compared with single-carrier modulation formats, DMT shows high flexibility to suit the non-flat response with adaptive bits-power loading technique. Besides, with the help of cyclic prefix it is less sensitive to the ISI. However, the main disadvantage is the relatively complex DSP required for DMT implementation. A short summary of state-of-the-art SDM enabled DCN links is presented in Table. 1. It can be seen that PAM4 shows a good trade-off among various performance metrics compared to NRZ/EDB and DMT. The choice of modulation formats in SDM based DCNs needs to consider the network and transmission requirements.

Digital signal processing (DSP) algorithm design for compensating impairments in SDM based DCN links is also of great importance. The implementation cost of DSP is decreasing rapidly by advancements in CMOS fabrication, and the powerful DSP that has already been employed in long-haul systems may gradually be applied in short-reach DCNs. The MIMO-DSP is a powerful tool to mitigate inter-mode crosstalk in SDM links, which can be divided into time-domain equalizer (TDE) and frequency-domain equalizer (FDE). The TDE based MIMO-DSP algorithm [83] always suffers from high computational complexity because of a large number of equalization memory taps. Dividing a single MIMO equalization into 'partial MIMO' equalizations [84] in which TDE is carried out for lower and higher order mode signals independently enables reduction of the TDE MIMO-DSP complexity while keeping optical SNR penalty low $(\sim 1 \mathrm{~dB})$. As an alternative, the use of FDE techniques [85-86] is prominent to mitigate the equalizer complexity. Nevertheless, the MIMO-DSP is not necessary when multimode transmission is deployed for DCNs where the reach is short [18].

It is shown in Section II that in uncoupled or weakly-coupled MCFs the IC-XT is rather small. Consequently, DSP is not needed there. However, DSP is still required to mitigate the IC-XT for strongly-coupled MCFs. In [87], the authors propose to use a time domain FFE based MIMO algorithm to compensate the performance penalty induced by IC-XT $(-4 \mathrm{~dB})$ in a strongly-coupled 6-core fiber. The feasibility of employing the MIMO algorithm for suppressing IC-XT needs to be further analyzed.

Table. 1. The comparison of state-of-the-art SDM enabled DCN links.

\begin{tabular}{|c|c|c|c|c|c|c|}
\hline Modulation & $\begin{array}{l}\text { Spectral } \\
\text { efficiency }\end{array}$ & $\begin{array}{l}\text { DSP } \\
\text { complex. }\end{array}$ & Flexibility & $\begin{array}{l}\text { SNR } \\
\text { req. }\end{array}$ & $\begin{array}{l}\text { Tolerance to } \\
\text { SDM crosstalk }\end{array}$ & $\begin{array}{l}\text { State-of-the-art data rate } \\
* \text { distance }\end{array}$ \\
\hline $\begin{array}{l}\text { NRZ/EDB } \\
3 \pm .19 \text {, } 80.8 \%\end{array}$ & Low & Low & Low & Low & Low & 700Gbps*10km (EML) [77] \\
\hline PAM4 & Medium & Medium & Low & High & Medium & $\begin{array}{l}\text { 1043Gbps*1km (EML) [78] } \\
\text { 700Gbps*10km (VCSEL) [81] }\end{array}$ \\
\hline 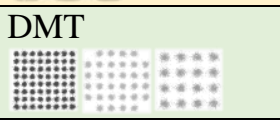 & High & High & High & High & Medium & $\begin{array}{l}\text { 726.6Gbps*2.5km (VCSEL) [24] } \\
533.1 \mathrm{Gbps} * 10 \mathrm{~km} \text { (VCSEL) [24] }\end{array}$ \\
\hline
\end{tabular}


Table. 2. A summary of main DSP equalization algorithms for SDM based DCN applications.

\begin{tabular}{l|l}
\hline Types of impairments & DSP algorithms \\
\hline \multirow{2}{*}{ Inter-mode XT } & TDE-MIMO [83, 84] \\
\cline { 2 - 2 } & FDE-MIMO [85, 86] \\
\hline IC-XT & TDE-MIMO [87] \\
\hline $\begin{array}{l}\text { Linear Impairments } \\
\text { (e.g. chromatic dispersion, } \\
\text { receiver noise) }\end{array}$ & LS [24] \\
\cline { 2 - 2 } $\begin{array}{l}\text { Nonlinear Impairments } \\
\text { (e.g. laser chirp, } \\
\text { amplifier saturations) }\end{array}$ & MLSE [88] \\
\cline { 2 - 2 } & Volterra filtering [24, 82] \\
\cline { 2 - 2 } & $\begin{array}{l}\text { KL [89] } \\
\text { kernel-LMS [90], }\end{array}$ \\
\hline
\end{tabular}

For high-speed SDM links working at long wavelength in DCNs, the main impairments include the linear impairments caused by dispersion, background noise from opto-electrical components and the nonlinear impairments, e.g. laser chirp and amplifier saturations [24]. To mitigate the linear impairments, linear adaptive filtering based equalizations (e.g. least-squares LS [24], FFE [77, 81], decision feedback equalization DFE [78, 81], and maximum likelihood sequence estimate MLSE [88]) work well. To mitigate the nonlinear impairments, nonlinear equalizations are required. The Volterra series based nonlinear equalization is a conventional scheme to compensate nonlinear impairments [24]. Besides, machine learning (ML) based nonlinear equalization is gaining a lot of interest [89]. It remains an open question whether the high computational complexity of ML-based equalizers is proper in SDM-based DCNs. Recently, we proposed to use kernel filtering scheme for nonlinear impairments compensation [90-91]. Kernel method is a mapping scheme, where Mercer kernels can be utilized to map the low-dimension signal into the high-dimension one. Such a mapping makes a low-complexity linear adaptive filtering mechanism possible for nonlinear equalization. Kernel-least-mean-squares (kernel-LMS) [90] and kernel-recursive-least-square (kernel-RLS) [91] are introduced. The results have demonstrated that kernel-LMS and kernel-RLS can compensate the nonlinear impairments in an effective way. A short summary of DSP algorithms is shown in Table. 2.

Nevertheless, with the increasing capacity requirement in DCNs, the debate on coherent and direct detection schemes is getting more attentions. For coherent detection, the main concern is the high cost of the transceiver and DSP. Thanks to the homogeneous spatial channels in SDM fibers, the signals after SDM DEMUX connecting different cores in one MCF suffer from similar link impairments at the receiver side, and the coherent MIMO processing complexity can be reduced by sharing the same equalizer mechanism among the signals transmitted over various cores in the MCF. Besides, by transmitting the local oscillator at transmitter side as pilot tone to be used at the receiver, the self-homodyne coherent detection scheme becomes less sensitive to the laser phase noise [92], and low-cost distributed feedback laser can be deployed. Moreover, the progress of coherent technique in SDM based short-reach communications makes FMF transmission that always needs coherent receivers [16] more practical to be employed in DCNs. Thus, employing coherent techniques for SDM based DCNs becomes interesting, which encourages research efforts on the corresponding transceiver design and DSP algorithms.

\section{Network Aspects: Architectures AND ResourCe ALLOCATION STRATEGIES}

Advances in SDM component and transmission techniques for short-reach applications provide foundations for research on network aspects, particularly on architecture and the resource allocation strategies for DCNs. Combining WDM and SDM to increase the capacity and flexibility may be a feasible approach for extending the conventional WDM with the space domain. There are several works that investigate different ways for supporting spectrally-spatially flexible optical long-haul networks, e.g., [93], where long wavelengths (the $\mathrm{C}$ band, $1.5 \mu \mathrm{m})$ are considered for the spectrum allocation. The traditional fixed-grid WDM paradigm at long wavelengths, where the center frequencies of all connections are uniformly spaced along the frequency axis, is here gradually being replaced by spectrally elastic channels with different bandwidths, so-called flexgrid [103-104]. As reviewed in previous sections, currently long wavelength techniques for both the components and transmission are still not a mainstream for DCNs, whereas this waveband may become essential in the future for the further capacity increase of DCNs. From the network perspective, introducing only SDM domain into switching in DCNs is straightforward (see Fig. 6a), having an advantage that there is no restriction for selecting a desired waveband. On the other hand, involving long wavelength techniques in DCNs in the future will bring a great potential to integrate SDM with fixed-grid WDM or flexgrid, although there are currently only few works addressing this opportunity in DCNs [14, 96-101].

The choice of the network topology has a significant impact on both the performance and the cost. In [14], different topologies (2D Torus, Star, Spin-Leaf, Facebook and Data Vortex) are investigated for the SDM-only with MCF in DCNs. Similar to the WDM-only scheme, the Spine-Leaf topology offers much higher improvement of throughput per cost unit (Gbps/cost unit) and throughput per unit power (Gbps/W) compared to all the other options. Topology-adaptive architectures [101] are also proposed to support the dynamically changing traffic patterns with the benefit of greatly simplifying the cabling and significantly reducing the number of required transceivers. Hereafter, we focus on architectural options and resource allocation strategies for DCNs, where so-called superchannels are formed by assigning multiple spatial and/or spectral resources to the same connection. Fig. 6 presents 5 architectural options. Their possible mapping to a multi-spatial element fiber using different SDM and spectrum allocation schemes can be similar as for long-haul networks [102]. Here, $N$ corresponds to the number of spatial elements per fiber and $M$ is the number of spectral slots per spatial channel. The spatial superchannel 
means the group of sub-channels occupying the same spectrum and routed together on a group of the spatial elements. On the other hand, the spectral superchannel refers to the group of sub-channels multiplexed on a separate spectral channel and routed together on the same spatial element.

The ungrouped SDM architecture (A1) employs SDM [97], where only spatial switching is available. The maximum number of channels that can be established per fiber is $N$. Resource allocation only needs to handle the spatial element allocation. The first-fit (FF) algorithm is a simple greedy scheduling algorithm for dynamic resource allocation, where for every new connection request, the first available spatial element is allocated. In [102], a proposed core priority algorithm is used as a pre-defined policy to reduce the IC-XT. Only if there are available resources and the IC-XT is below the pre-set threshold, the request will be accepted. Using such an
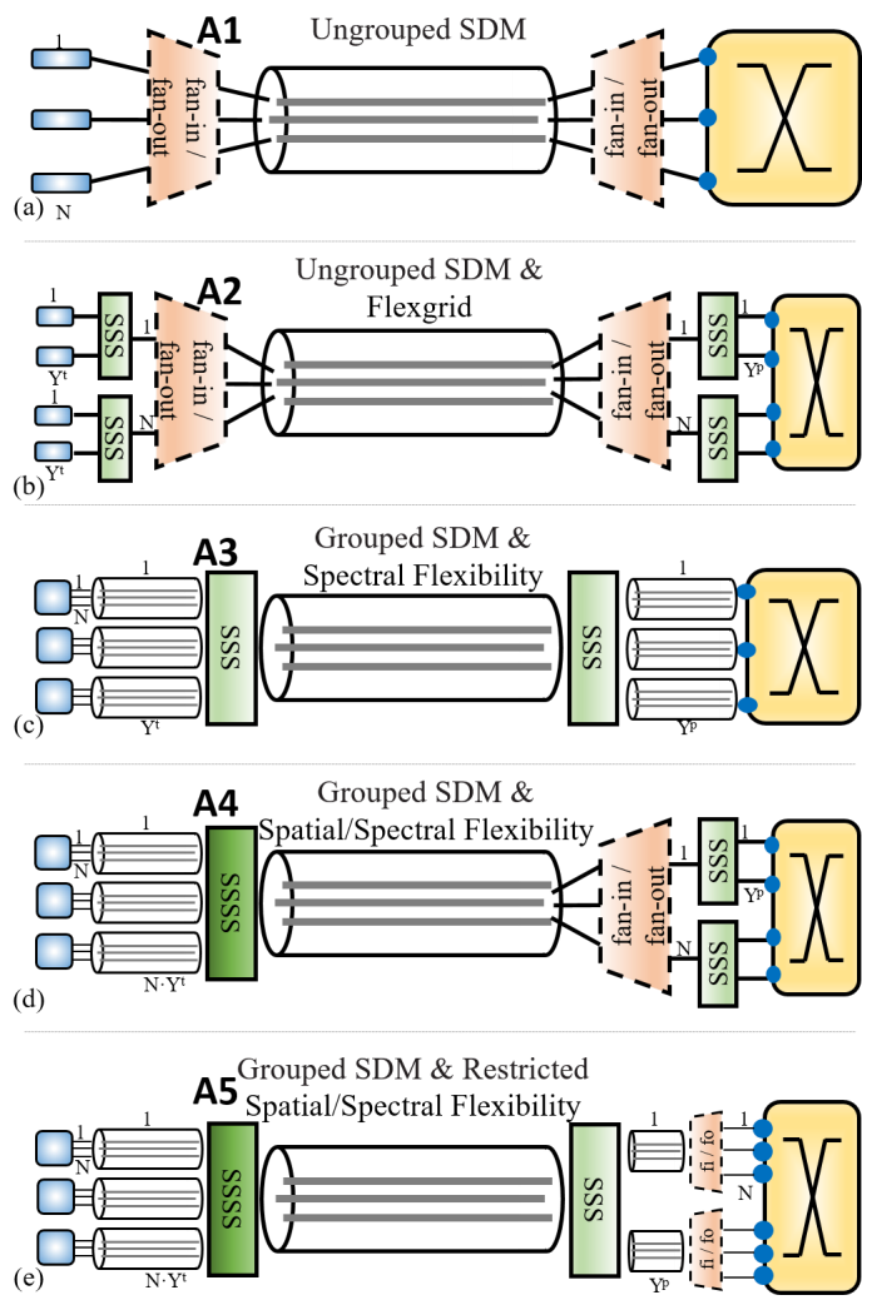

Fig. 6. SDM based DCN architectural options (left) of (a) A1: ungrouped SDM, (b) A2: ungrouped SDM and flexgrid, (c) A3: grouped SDM with spectral flexibility, (d) A4: grouped SDM with spectral and spatial flexibility and (e) A5: grouped SDM with restricted spectral and spatial flexibility. algorithm, the ungrouped SDM based solution (A1) has comparable performance with the WDM-only scheme in terms of network capacity per cost and per power consumption. An alternative of $\mathrm{A} 1$ is to expand flexibility in the spatial domain, which corresponds to the grouped SDM architecture with fixed grid WDM. In such architecture, a superchannel can be transmitted on several spatial elements occupying one frequency grid on every spatial element. However, this scheme offers limited capacity improvement because of the unused resources caused by the fact that one assigned superchannel needs to occupy the allocated spectrum resources on all the spatial elements [102].

The ungrouped SDM and flexgrid architecture (A2) [97] allows each spatial element to work as a separate flexgrid transmission medium. Similarly to A1, in this option each spatial element is independent. The number of channels that can be established per fiber is at most $M \cdot N$. The architecture requires $2 N$ spectrum selective switches (SSSs) and two spatial fan-in/fan-out per server pod (referred to as an atomic unit in DCNs including computation, networking and storage resources [97, 103]), see Fig. 6b. Resource allocation needs to be done in both spatial and spectral domains. When employing the FF algorithm, for every new connection request the spatial elements and spectral slots are checked iteratively and the iteration is stopped once a sufficient number of available contiguous spectral slots are found.

The grouped SDM with spectral flexibility architecture (A3) [97-99], see Fig. 6c, expands spectral superchannels in the spatial domain to create spectral-spatial superchannels with spatial coupling, where MIMO optical transceivers are used. In Fig. 6c, the employment of two large SSSs is considered. Such a structure can also be replaced by combining SDM MUX/DEMUX and WSS as proposed in [98-99], where the corresponding resource allocation for one connection requires the same spectral slots assigned in different spatial elements. Although A3 exploits the spatial dimension to create spectral superchannels, the flexibility is limited. The maximum number of channels supported by a single fiber is determined by $M$.

The grouped SDM with spectral and spatial flexibility architecture (A4) has unrestricted flexibility in both the spectral and spatial domains, which leads to the highest possible degree of flexibility. The number of channels that can be established per fiber in this case is at most $M \cdot N$. On the other hand, A4 requires a large spectral and spatial selective switch (SSSS) to connect each server pod to the multi-spatial element fiber. Because of the high flexibility, advanced resource allocation approaches rather than $\mathrm{FF}$ are required to ensure efficient resource utilization. In [104], it is demonstrated that the 'spectrum resources first' (SpeF) algorithm is more efficient than the 'spatial resources first' (SpaF). Therefore, it is recommended to accommodate each new connection request using a spectral superchannel first and if it fails to find free resources, spectral-spatial superchannels are created by increasing the number of the allocated spatial elements.

The grouped SDM with restricted spectral and spatial flexibility architecture (A5) [97, 100] allows to establish flexible spectral-spatial superchannels, but needs to make sure 
that the spectral-spatial superchannels belonging to the same spectral group utilize the same spectral resources. An SSSS is required to connect each server pod to the SDM fiber. This restricted flexibility reduces the complexity of the resource allocation compared with A4. To some degree, A5 extends A3. The number of parallel channels that can be established over the same fiber is lower than $M \cdot N$. The spectral group constraint in A5 makes the SpeF strategy less efficient and thus the SpaF strategy can be employed to maximize the use of the spatial resources. If it fails to find free spectral resources on any of the spatial elements, the spectral-spatial superchannels are created using an increasing number of spectral slots until a feasible solution is found.

Comparing the aforementioned architectures for SDM based DCNs, the more efficient resource utilization reflected by the lower blocking probability of the architecture can be achieved by the higher resource allocation flexibility [97-99]. However, when the network cost is considered. A1 represents the best option for a relatively small DCN (e.g., private enterprise DCNs), A3 is the best option for a medium DCN (e.g., medium-sized cloud provider), and $\mathrm{A} 2$ is the best option for large cloud provider [97]. When the load is high, A4 is the only viable solution for a very large DCN. It can be seen that introducing SDM to DCNs is very beneficial from the network architecture point of view. For small-size DCNs, employing purely SDM is sufficient. When the load is high and/or the size is large, combining SDM with flexgrid is helpful, but the unrestricted flexibility based on the state-of-the-art technology is not cost-efficient in most of the cases.

Meanwhile, IC-XT is one of the constraints for the core and spectrum resource allocation due to its impact on the transmission quality. The transmission quality degradation can be reduced by heterogeneous MCF deployment, in which IC-XT can be significantly lowered. It can be also done by allocating bi-directional propagation of signals in the neighboring cores or properly selecting the sequence of the core usage [14]. The dynamic spectrum and core allocation method that reduces both the crosstalk and fragmentation in elastic optical networks with MCFs was proposed in [104, 106]. Taking IC-XT into account, integer linear programming (ILP [105-106]) and mixed integer linear programming (MILP [107]) formulation as well as heuristic algorithms for resource allocation and routing strategies in SDM network are well studied, targeting minimization of the blocking probability [107-109] and maximization of the total throughput [104, 106], which can be generalized for DCN applications with a shorter transmission distance. The detailed trade-off between the throughput and blocking probability in SDM-based DCs is investigated in [110].

Nevertheless, there are still some technical challenges to be solved considering network aspects in SDM based DCNs. Currently, most of studies on the combination of SDM and WDM for DCNs do not consider latency and power consumption, which are of key importance for DCNs. The introduction of spectrum switching function into SDM based DCNs may bring extra latency and power consumption compared with the pure SDM solutions. Combining WDM and
SDM switching may also lead to a higher level of transmission impairments due to the filtering and power loss, which requires extensive use of DSP or high power transceivers. Consequently, it may result in a significant increase on power consumption and latency. Therefore, the research towards a comprehensive comparison in terms of scalability, power consumption, switching and processing latency needs to be carried out in the future to address these questions.

Recently, the DC disaggregation was introduced as a novel paradigm to improve the resource utilization in DCNs [111]. For rack-scale disaggregation, the integrated server blades containing all types of resources are replaced by the resource blades including only one specific function. However, the communication between different resource blades faces severe problems in terms of latency and transmission bandwidth. The peak bandwidth requirement of the CPU and memory communication is 500Gbps and beyond, while the latency should be kept below 100ns. Obviously, SDM transmission has a great potential to address capacity requirement for the DC disaggregation. In the recently proposed resource centric disaggregated data center architecture [112], the integration of MCF-based transceivers, fibers, and optical switches is experimentally validated. The results show the benefits of SDM in terms of capacity, power consumption, latency and space efficiency for interconnecting disaggregated IT elements. To support function disaggregation in DCs, SDM will be most probably first employed within the rack for ultra-short distance interconnects. On the other hand, core-tier switches in large-scale DCs handle the aggregated traffic flows and hence also require high capacity, which may motivate to employ SDM there as well.

\section{SUMmary AND CONCLUSIONS}

Compared to parallel optical fibers or fiber bundles/ribbons, MCF, FMF, and a combination of both have shown a great potential to improve the spatial efficiency for future DCNs. A straightforward way to realize switching granularity on a per-spatial-channel basis is to place SDM MUX/DEMUX modules at the ports of switches, for which integrated circuits using MEMS, LCoS, or silicon photonics are candidate techniques. From the transceiver point of view, a VCSEL array has the advantages of simple integration with MCF and low cost. Nevertheless, EML, DML and MZM might be considered in the near future thanks to high bandwidth. Multiple modulation formats should be considered, as single-carrier and multicarrier formats display tradeoffs in terms of spectral efficiency, flexibility, SNR requirements, and tolerance to crosstalk in SDM-based DCNs. DSP is a versatile and powerful technology to compensate both linear and nonlinear impairments, which will be introduced gradually in future DCNs as capacity requirements increase and the cost of electronics decreases. Introducing SDM to DCNs is also beneficial from a network architecture point of view. For small-size DCNs, pure SDM is sufficient. When the load and/or size are scaled up, applying SDM combined with WDM may be helpful. Physical layer impairments cannot be ignored for network architecture design and resource allocation. 
Table. 3. A summary of the key SDM technologies for DCNs.

\begin{tabular}{|c|c|c|c|}
\hline \multicolumn{3}{|c|}{$\begin{array}{l}\text { SDM technologies for component, transmission and } \\
\text { networking aspects }\end{array}$} & \multirow{2}{*}{\begin{tabular}{|l|} 
Technical features \\
Commercially deployed \\
Not spatially efficient enough \\
Low potential of photonics integration
\end{tabular}} \\
\hline \multirow{16}{*}{$\begin{array}{l}\text { SDM } \\
\text { Components }\end{array}$} & \multirow{6}{*}{ Fibers } & $\begin{array}{l}\text { Parallel fibers } \\
\text { Fiber bundles/ribbons }\end{array}$ & \\
\hline & & Multi-core fiber (MCF) & $\begin{array}{l}\text { High spatial efficiency } \\
\text { Low IC-XT (un-coupled/weakly-coupled) } \\
\text { No MIMO-DSP (un-coupled/weakly-coupled) }\end{array}$ \\
\hline & & $\begin{array}{l}\text { Few-mode fiber (FMF): A simpler version of } \\
\text { multi-mode fiber (MMF) }\end{array}$ & $\begin{array}{l}\text { Medium-high spatial efficiency } \\
\text { Inter-mode crosstalk } \\
\text { Needs MIMO-DSP } \\
\text { SDM in MMF has not yet been fully explored }\end{array}$ \\
\hline & & Few-mode multi-core fiber (FM-MCF) & $\begin{array}{l}\text { High spatial efficiency } \\
\text { Medium inter-core and inter-mode crosstalk } \\
\text { Limited MIMO-DSP } \\
\end{array}$ \\
\hline & & Elliptical core few-mode fiber & $\begin{array}{l}\text { Medium-high spatial efficiency } \\
\text { Low crosstalk } \\
\text { No MIMO-DSP }\end{array}$ \\
\hline & & Hollow-core fiber & $\begin{array}{l}\text { Medium-high spatial efficiency } \\
\text { Higher light speed } \\
\text { Mechanical precision requirements } \\
\text { High loss }\end{array}$ \\
\hline & \multirow{4}{*}{$\begin{array}{l}\text { MCF fan-in/ } \\
\text { fan-out }\end{array}$} & Free space optics & $\begin{array}{l}\text { Tolerant to the offset of the core position } \\
\text { Sophisticated opto-mechanical operations }\end{array}$ \\
\hline & & Fused taper & Compact and cost-efficient fabrication process \\
\hline & & Fiber bundle & XT needs to be carefully controlled \\
\hline & & Compact waveguide coupling & High potential of photonics integration \\
\hline & \multirow{3}{*}{$\begin{array}{l}\text { FMF/MMF } \\
\text { MUX/DEM } \\
\mathrm{UX}\end{array}$} & Mode conversion & High conversion and combination loss \\
\hline & & Index matching & Complex fabrication process \\
\hline & & Photonics lantern & Similar as fan-in/fan-out but higher spatial density \\
\hline & \multirow{3}{*}{ Switches } & Micro electro mechanical systems mirror (MEMS) & Steady hardware complexity \\
\hline & & Liquid crystal on silicon (LCOS) & Precise opto-mechanical operations \\
\hline & & Silicon photonic integrated circuit & High potential of photonics integration \\
\hline \multirow{9}{*}{$\begin{array}{l}\text { Transmissio } \\
\mathrm{n} \text { Aspects }\end{array}$} & \multirow{3}{*}{ Transceivers } & Short wavelength VCSEL & $\begin{array}{l}\text { Ease of integrated into array } \\
\text { Low cost and power consumption, short-reach }\end{array}$ \\
\hline & & Long wavelength VCSEL & $\begin{array}{l}\text { Ease of integrated into array } \\
\text { Low cost and power consumption, longer-reach } \\
\text { Compatibility with coarse or dense WDM } \\
\end{array}$ \\
\hline & & DML, EML, MZM & $\begin{array}{l}\text { High bandwidth } \\
\text { Comparatively high cost for SDM parallel transmission }\end{array}$ \\
\hline & \multirow{3}{*}{ Modulation } & Simple single carrier (e.g. NRZ) & $\begin{array}{l}\text { Simple and ease of implementation } \\
\text { Low spectrum efficiency } \\
\text { High tolerance to noise and IC-XT }\end{array}$ \\
\hline & & Advanced single carrier (e.g. PAM4) & $\begin{array}{l}\text { Ease of implementation } \\
\text { Medium spectrum efficiency } \\
\text { Medium tolerance to noise and IC-XT } \\
\end{array}$ \\
\hline & & Multicarrier (e.g. DMT) & $\begin{array}{l}\text { High implementation cost } \\
\text { High spectrum efficiency and flexibility } \\
\text { Medium tolerance to IC-XT }\end{array}$ \\
\hline & \multirow{3}{*}{ DSP } & FDE-MIMO, TDE-MIMO & MIMO-DSP compensating inter-mode crosstalk and IC-XT \\
\hline & & LS, FFE, DFE, MLSE & Compensating linear impairments (e.g. dispersion) \\
\hline & & Volterra, Kernel & Compensating nonlinear impairments (e.g. VCSEL chirp) \\
\hline \multirow{5}{*}{$\begin{array}{l}\text { Network } \\
\text { Aspects }\end{array}$} & \multirow{5}{*}{$\begin{array}{l}\text { Architecture } \\
\& \\
\text { Resource } \\
\text { allocation }\end{array}$} & A1: Ungrouped SDM with FF spatial allocation & Suitable for a small DCN (e.g., private enterprise DCNs) \\
\hline & & $\begin{array}{l}\text { A2: Ungrouped SDM and flexgrid with FF spatial } \\
\text { and spectral allocation }\end{array}$ & Suitable for a large cloud provider \\
\hline & & $\begin{array}{l}\text { A3: Grouped SDM with spectral flexibility } \\
\text { spectral allocation }\end{array}$ & $\begin{array}{l}\text { Suitable for a medium DCN (e.g., medium-sized cloud } \\
\text { provider) }\end{array}$ \\
\hline & & $\begin{array}{l}\text { A4: Grouped SDM with spectral and spatial } \\
\text { flexibility with spectrum resources first (SpeF) }\end{array}$ & Only viable solution for a very large DCN or with a high load \\
\hline & & $\begin{array}{l}\text { A5: Grouped SDM with restricted spectral and } \\
\text { spatial flexibility with spatial resources first } \\
\text { (SpaF) }\end{array}$ & $\begin{array}{l}\text { Extends A3 by improving throughput, but suffers from high } \\
\text { cost }\end{array}$ \\
\hline
\end{tabular}


Based on the state-of-the-art SDM technologies employed in DCNs, the key technologies and their technical features are summarized in Table 3, including components, transmission, and network aspects of SDM-based DCNs. While SDM serves as an enabler for future DCNs with ultra-high capacity, a joint optimization including component, transmission and network aspects still requires substantial research efforts.

Regarding the components, low crosstalk SDM fibers with higher spatial efficiency, such as increased core and/or mode count in a single fiber, are expected to fulfill the future capacity boost in DCNs. The pluggable SDM transceivers will be beneficial for deployment of SDM techniques. Besides, core-to-core SDM switches with low IC-XT and insertion loss could be promising for rack-scale switching in disaggregated data centers. Considering the transmission aspect, a comprehensive comparison of direct detection and coherent detection could be very useful to provide a guideline for selecting proper detection techniques for SDM based DCNs. Self-homodyne coherent detection shows a high potential to increase the DCNs capacity while maintaining a low DSP overhead and high transmission quality, but a further validation is still required. Besides, the IC-XT fluctuations need to be considered for DCN links, where time-domain adaptive DSP algorithms have a great potential to mitigate the impairments that vary in time. The IC-XT fluctuations need also be considered on network layer, where impairment-aware resource allocation strategies are highly demanded for SDM based DCNs.

Last but not least, SDM techniques need to convince service providers and network vendors that they are beneficial, in order to be deployed in the DCN infrastructure. Therefore, more research on the flexible DCN architecture and resource allocation strategies tailored for the deployed SDM techniques are expected. Apart from capacity, the other key metrics such as cost, power consumption, latency and reliability will be of key importance to be considered for SDM based DCNs.

\section{ACKNOWLEDGMENTS}

We thank Xiaodan Pang from Infinera, Sweden with discussion on the contents in Section. III. A, and Aleksejs Udalcovs and Oskars Ozolins, from RISE, Sweden, with discussion on the contents in Section. III. B.

\section{REFERENCES}

[1] J. Clark, "The rise of mega data centers," The Data Center Journal, May. 2012.

[2] https://www.datacenterknowledge.com/

[3] Cisco global cloud index: forecast and methodology, 2016-2021. https://www.cisco.com/c/en/us/solutions/collateral/service-provider/globa 1-cloud-index-gci/white-paper-c11-738085.html

[4] Cisco visual networking index: forecast and trends, 2017-2022. https://www.cisco.com/c/en/us/solutions/collateral/service-provider/visual -networking-index-vni/white-paper-c11-741490.html

[5] A. S. G. Andrae, and T. Edler, "On global electricity usage of communication technology: trends to 2030," Challenges, vol. 6, no. 1, pp. 117-157, Apr. 2015.

[6] NGMN 5G white paper.

https://www.ngmn.org/5g-white-paper/5g-white-paper.html

[7] Coriant Blog, "Evolution of the data center interconnect ecosystem," 2016. http://www.coriant.com/blog/2016/05/12/evolution-of-the-data-center-int erconnect-dci-ecosystem/

[8] C. Kachris, K. Bergman, and I. Tomkos, "Optical interconnects for future data center networks," Springer Science \& Business Media, 2012.

[9] IEEE Standard, Media access control parameters, physical layers, and management parameters for $200 \mathrm{~Gb} / \mathrm{s}$ and $400 \mathrm{~Gb} / \mathrm{s}$ operation.

[10] M. Li, "New development trends in optical fibers for data centers," in Proc. ECOC, Rome, Sep. 2018.

[11] X. Zhou, R. Urata, and H. Liu, "Beyond $1 \mathrm{~Tb} / \mathrm{s}$ datacenter interconnect technology: challenges and solutions," in Proc. OFC, San Diego, Tu2F.5, Mar. 2019.

[12] L. Zhang, X. Hong, X. Pang, O. Ozolins, A. Udalcovs, R. Schatz, C. Guo, J. Zhang, F. Nordwall, K. M. Engenhardt, U. Westergren, S. Popov, G. Jacobsen, S. Xiao, W. Hu, and J. Chen, "Nonlinearity-aware $200 \mathrm{Gbit} / \mathrm{s}$ DMT transmission for C-band short-reach optical interconnects with a single packaged electro-absorption modulated laser," Opt. Lett., vol. 43, no.2, pp. 182-185, Jan. 2018.

[13] P. J. Winzer, "Making spatial multiplexing a reality," Nat. Photon., vol. 8, no. 5, pp. 345-348, May 2014.

[14] Y. Liu, H. Yuan, A. Peters, and G. Zervas, "Comparison of SDM and WDM on direct and indirect optical data center networks," in Proc. ECOC, Dusseldorf, Germany, Sep. 2016.

[15] C. Kachris, and I Tomkos, "A survey on optical interconnects for data centers," IEEE Com. Surveys Tuts., vol. 14, no. 4, pp. 1021-1036, Oct. 2012.

[16] G. M. Saridis, D. Alexandropoulos, G. Zervas, and D. Simeonidou, "Survey and evaluation of space division multiplexing: from technologies to optical networks," IEEE Com. Surveys Tuts., vol. 17, no. 4, pp. 2136-2156, Dec. 2015.

[17] A. Singh, J. Ong, A. Agarwal, G. Anderson, A. Armistead, R. Bannon, S. Boving, G. Desai, B. Felderman, P. Germano, and A. Kanagala, "Jupiter rising: A decade of clos topologies and centralized control in Google's datacenter network," in Proc. SIGCOMM, London, pp. 183-197, Aug. 2015.

[18] B. Zhu, "SDM fibers for data center applications," in Proc. OFC, San Diego, M1F.4, Mar., 2019.

[19] M. Tang, "Multicore fibers," Handbook of Optical Fibers, 2018.

[20] D. J. Richardson, J. M. Fini, and L. E. Nelson, "Space-division multiplexing in optical fibers," Nat. Photon., vol. 7, no. 5, pp. 354-362, May 2013.

[21] W. Klaus, B. J. Puttnam, R. S. Luís, J. Sakaguchi, J. M. Delgado Mendinueta, Y. Awaji, and N. Wada, "Advanced space division multiplexing technologies for optical networks," J. Opt. Commun. Netw., vol. 9, no. 4, pp. C1-C11, Apr. 2017.

[22] K. Takenaga, Y. Arakawa, S. Tanigawa, N. Guan, S. Matsuo, K. Saitoh, and M. Koshiba, "Reduction of crosstalk by trench-assisted multi-core fiber," in Proc. OFC, Los Angeles, OWJ4, Mar. 2011.

[23] K. Saitoh, and S. Matsuo, "Multicore fiber technology," J. Lightw. Technol., vol. 34, no. 1, pp. 55-66, Jan. 2016.

[24] L. Zhang, J. Van Kerrebrouck, R. Lin, X. Pang, A. Udalcovs, O. Ozolins, S. Spiga, MC Amann, G. Van Steenberge, L. Gan, M. Tang, S. Fu, R. Schatz, S. Popov, D. Liu, W. Tong, S. Xiao, G. Torfs, J. Bauwelinck, X. Yin, and J. Chen, "Nonlinearity tolerant high-speed DMT transmission with $1.5-\mu \mathrm{m}$ single-mode VCSEL and multi-core fibers for optical interconnects," $J$. Lightw. Technol., vol. 37, no. 2, pp. 380-388, Jan. 2019.

[25] S. Matsuo, K. Takenaga, Y. Arakawa, Y. Sasaki, S. Taniagwa, K. Saitoh, and M. Koshiba, "Large-effective-area ten-core fiber with cladding diameter of about $200 \mu \mathrm{m}$," Opt. Lett., vol. 36, no. 23, pp. 4626-4628, Dec. 2011.

[26] S. Takasaka, H. Matsuura, W. Kumagai, and M. Tadakuma, "Cladding-pumped seven-core EDFA using a multimode pump light coupler," in Proc. ECOC, London, Sep. 2013.

[27] F. Ye, K. Saitoh, H. Takara, R. Asif, and T. Morioka, "High-count multi-core fibers for space-division multiplexing with propagation-direction interleaving," in Proc. OFC, Mar. 2015.

[28] D. L. Butler, M. Li, S. Li, Y. Geng, R. R. Khrapko, R. A. Modavis, V. N. Nazarov, and A. V. Koklyushkin, "Space division multiplexing in short reach optical interconnects," J. Lightw. Technol., vol. 35, no. 4, pp. 677-682, Feb. 2017.

[29] ITU-T G.657 A1 Standard.

[30] R. S. Luís, B. J. Puttnam, G. Rademacher, W. Klaus, E. Agrell, Y. Awaji, 
and N. Wada, "On the spectral efficiency limits of crosstalk-limited homogeneous single-mode multi-core fiber systems," in Proc. Advanced Photonics Congress, New Orleans, Jul. 2017.

[31] G. Rademacher, R. S. Luís, B. J. Puttnam, Y. Awaji, and N. Wada, "Crosstalk dynamics in multi-core fibers," Opt. Exp., vol. 25, no. 10, pp. 12020-12028, 2017.

[32] F. Ye, J. Tu, K. Saitoh, K. Takenaga, S. Matsuo, H. Takara, and T. Morioka, "Wavelength-dependence of inter-core crosstalk in homogeneous multi-core fibers," Photon. Technol. Lett., vol. 28, no. 1, pp. 27-30, Jan. 2016.

[33] H. Yuan, A. Saljoghei, T. Hayashi, T. Nakanishi, E. Sillekens, L. Galdino, P. Bayvel, Z. Liu, and G. Zervas, "Experimental investigation of static and dynamic crosstalk in trench assisted multi-core fiber," in Proc. OFC, San Diego, W4C.2, Mar. 2019.

[34] R. S. Lu'is, B. J. Puttnam, A. V. T. Cartaxo, W. Klaus, J. M. D. Mendinueta ', Y. Awaji, N. Wada, T. Nakanishi, T. Hayashi, and T. Sasaki, "Time and modulation frequency dependence of crosstalk in homogeneous multi-core fibers," J. Lightw. Technol., vol. 34, no. 2, pp. 441-447, Jan. 2016.

[35] G. Rademacher, R. S. Luís, , B. J. Puttnam, Y. Awaji, and N. Wada, "Modulation format-dependence of crosstalk fluctuations in homogeneous multi-core fibers," in Proc. CLEO, Munich, Jun. 2017.

[36] R. Ryf, and M. K. Fontaine, "Space-division multiplexing and MIMO processing," in Enabling Technologies for Optical Data Center Networks: Spatial Division Multiplexing. X. Zhou, and C. Xie, Eds. Wiley, Ch. 16, 2016.

[37] A. Udalcovs, R. Lin, O. Ozolins, L. Gan, L. Zhang, X. Pang, R. Schatz, A. Djupsjöbacka, M. Tang, S. Fu, D. Liu, W. Tong, S. Popov, G. Jacobsen, and J. Chen, "Inter-core crosstalk in multicore fibers: impact on 56-Gbaud/ $\lambda$ /core PAM-4 transmission," in Proc. ECOC, Rome, Sep. 2018.

[38] M. Bigot-Astruc, D. Boivin, and P. Sillard, "Design and fabrication of weakly-coupled few-modes fibers," in Proc. IEEE Photon. Soc. Summer Topical Meet. Ser., pp. 189-190, Seattle, Aug. 2012.

[39] M.-J. Li, "New development trends in optical fibers for data centers," in Proc. ECOC, Rome, Sep. 2018.

[40] E. Ip, G. Milione, M. Li, N. Cvijetic, K. Kanonakis, J. Stone, G. Peng, X. Prieto, C. Montero, V. Moreno, and J. Liñares, "SDM transmission of real-time $10 \mathrm{GbE}$ traffic using commercial SFP + transceivers over $0.5 \mathrm{~km}$ elliptical-core few-mode fiber", Opt. Exp., vol. 23, no. 13, pp. 17120-17126, 2015.

[41] V. Sleiffer, Y. Jung, N. Baddela, J. Surof, M. Kuschnerov, V. Veljanovski, J. Hayes, N. Wheeler, E. Numkam Fokoua, J. Wooler, D. Gray, N. Wong, F. Parmigiani, S. Alam, M. Petrovich, F. Poletti, D. Richardson, and H. de Waardt, "High capacity mode-division multiplexed optical transmission in a novel 37-cell hollow-core photonic bandgap fiber," J. Lightw. Technol., vol. 32, no. 4, pp. 854-863, Feb., 2014.

[42] V. Mikhailov, J. M. Fini, L. Meng, B. J. Mangan, J. W. Nicholson, R. S. Windeler, E. Monberg, F. DiMarcello, and P. S. Westbrook, "Low-loss low-latency transmission over single- mode hollow core fiber at 10 and $120 \mathrm{~Gb} / \mathrm{s}$," in Proc. OFC, San Francisco, Mar. 2014.

[43] Y. Tottori, T. Kobayashi, and M. Watanabe, "Low loss optical connection module for seven-core multicore fiber and seven single-mode fibers," Photon. Technol. Lett., vol. 24, no. 21, pp. 1926-1928, Nov. 2012.

[44] H. Uemura, K. Omichi, K. Takenaga, S. Matsuo, K. Saitoh, and M. Koshiba, "Fused taper type fan-in/fan-out device for 12 core multi-core fiber," in Proc. OECC, Melbourne, Jul. 2014.

[45] Y. Abe, K. Shikama, S. Yanagi, and T. Takahashi, "Low-loss physical-contact-type fan-out device for 12-core multicore fiber," in Proc. ECOC, London, Sep. 2013.

[46] T. Watanabe, M. Hikita, and Y. Kokubun, "19-core fan-in/fan-out waveguide device for dense uncoupled multi-core fiber," in Proc. IPC, Bellevue, Sep. 2013.

[47] OptoQuest, https://www.optoquest.co.jp/

[48] Optoscribe, www.optoscribe.com/

[49] Chiral Photonics, https://www.chiralphotonics.com/

[50] R. Ryf, S. Randel, A. H. Gnauck, C. Bolle, A. Sierra, S. Mumtaz, M. Esmaeelpour, E. C. Burrows, R.-J. Essiambre, P. J. Winzer, D. W. Peckham, A. H. McCurdy, and R. Lingle, Jr., "Mode-division multiplexing over $96 \mathrm{~km}$ of few-mode fiber using coherent $6 \times 6$ MIMO processing," $J$. Lightw. Technol., vol. 30, no. 4, pp. 521-531, Feb. 2012.

[51] G. Labroille, B. Denolle, P. Jian, P. Genevaux, N. Treps, and J.-F. Morizur,
"Efficient and mode selective spatial mode multiplexer based on multiplane light conversion," Opt. Exp., vol. 22, no. 13, pp. 15599-15607, Jun. 2014.

[52] A. M. J. Koonen, H. Chen, H. P. A. van den Boom, and O. Raz, "Silicon photonic integrated mode multiplexer and demultiplexer," Photon. Technol. Lett., vol. 24, no. 21, pp. 1961-1964, Nov. 2012.

[53] K. J. Park, K. Y. Song, Y. K. Kim, and B. Y. Kim, "All-fiber mode division multiplexer optimized for C-band," in Proc. OFC, San Francisco, Paper M3K.2, Mar. 2014.

[54] H. Kubota, M. Oguma, and H. Takara, "Three-mode multi/demultiplexing experiment using PLC mode multiplexer and its application to $2+1$ mode bi-directional optical communication," IEICE Electron. Exp., vol. 10, no. 12, pp. 1-6, Jun. 2013.

[55] S. G. Leon-Saval, N. K. Fontaine, J. R. Salazar-Gil, B. Ercan, R. Ryf, and J. Bland-Hawthorn, "Mode-selective photonic lanterns for space-division multiplexing," Opt. Exp., vol. 22, no. 1, pp. 1036-1044, Jan. 2014

[56] D. M. Marom, P. D. Colbourne, A. D’Errico, N. K. Fontaine, Y. Ikuma, R. Proietti, L. Zong, J. M. Rivas-Moscoso, I. Tomkos, "Survey of photonic switching architectures and technologies in support of spatially and spectrally flexible optical networking [Invited]," J. Opt. Commun. Netw., vol. 9, no. 1, pp. 1-26, Jan., 2017.

[57] L. E. Nelson, M. D. Feuer, K. Abedin, X. Zhou, T. F. Taunay, J. M. Fini, B Zhu, R. Isaac, R. Harel, G. Cohen, and D. M. Marom, "Spatial superchannel routing in a two-span ROADM system for space division multiplexing," J. Lightw. Technol., vol. 32, no. 4, pp. 783-789, Feb. 2014.

[58] N. K. Fontaine, T. Haramaty, R. Ryf, H. Chen, L. Miron, L. Pascar, M. Blau, B. Frenkel, L. Wang, Y. Messaddeq, S. LaRochelle, R. J. Essiambre, Y. Jung, Q. Kang, J. K. Sahu, S. U. Alam, D. J. Richardson, and D. M. Marom, "Heterogeneous space-division multiplexing and joint wavelength switching demonstration," in Proc. OFC, Mar. 2015.

[59] D. M. Marom, J. Dunayevsky, D. Sinefeld, M. Blau, R. Ryf, N. K. Fontaine, M. Montoliu, S. Randel, C. Liu, B. Ercan, M. Esmaeelpour, S. Chandrasekhar, A. H. Gnauck, S. G. Leon-Saval, J. Bland-Hawthorn, J. R. Salazar-Gil, Y. Sun, L. Grüner-Nielsen, and R. Lingle, Jr., "Wavelength-selective switch with direct few mode fiber integration," Opt. Exp., vol. 23, no. 5, pp. 5723-5737, Feb. 2015.

[60] R Wang, Q Wu, M Tang, S Fu, and D Liu, "Reconfigurable inter-core switching within multicore fiber," in Proc. OFC, San Diego, Mar. 2018.

[61] Y. Ding, V. Kamchevska, K. Dalgaard, F. Ye, R. Asif, S. Gross, M. J. Withford, M. Galili, T. Morioka, and L. K. Oxenløwe, "Reconfigurable SDM switching using novel silicon photonic integrated circuit," Scientific Reports, vol. 6, pp. 39058, 2016.

[62] H. C. H. Mulvad, A. Parker, B. King, D. Smith, M. Kovacs, S. Jain, J. Hayes, M. Petrovich, D. J. Richardson, and N. Parsons, "Beam-steering all-optical switch for multi-core fibers," in Proc. OFC, Los Angeles, Tu2C.4, Mar. 2017.

[63]C. Deakin, M. Enrico, N. Parsons, and G. Zervas, "Design and analysis of beam steering multicore fiber optical switches," J. Lightw. Technol., vol. 37, no. 9, pp. 1954-1963, May. 2019.

[64] A. N. Dames, "Beam-steering optical switch," US Patent 7389016, Jun. 2008.

[65] G. Li, N. Bai, N. Zhao, and C. Xia, "Space-division multiplexing: the next frontier in optical communication," Advances in Optics and Photonics, vol. 6, no. 4, pp. 413-487, 2014.

[66] Broadcom Inc., https://www.broadcom.com/

[67] Santec, www.santec.com/

[68] C. Kottke, C. Caspar, V. Jungnickel, R. Freund, M. Agustin, and N. N. Ledentsov, "High speed $160 \mathrm{~Gb} / \mathrm{s}$ DMT VCSEL transmission using pre-equalization," in Proc. OFC, Los Angeles, Mar. 2017.

[69] S. Spiga, W. Soenen, A. Andrejew, D. M. Schoke, X. Yin, J. Bauwelinck, G. Boehm, and M. C. Amann, "Single-mode high-speed 1.5- $\mu \mathrm{m}$ VCSELs," J. Light. Technol., vol. 35, no. 4, pp. 727-733, Aug. 2017.

[70] X. Yin, M. Verplaetse, L. Breyne, J. Van Kerrebrouck, T. De Keulenaer, A. Vyncke, R. Pierco, R. Vaernewyck, S. Spiga, M. -C. Amann, J. Chen, G. Van Steenberge, G. Torfs, and J. Bauwelinck, "Towards efficient $100 \mathrm{~Gb} / \mathrm{s}$ serial rate optical interconnects: A duobinary way," in Proc. OI, Santa Fe, NM, USA, Jun. 2017.

[71] C. Xie, P. Dong, S. Randel, D. Pilori, P. Winzer, S. Spiga, B. Kögel, C. Neumeyr, M.C. Amann, "Single-VCSEL 100-Gb/s short-reach system using discrete multi-tone modulation and direct detection," in Proc. OFC, Los Angeles, CA, USA, Mar. 2015. 
[72] N. Eiselt, H. Griesser, J. Wei, R. Hohenleitner, A. Dochhan, M. Ortsiefer, M. H. Eiselt, C. Neumeyr, J. J. V. Olmos, and I. T. Monroy, "Experimental demonstration of $84 \mathrm{~Gb} / \mathrm{s}$ PAM-4 over up to $1.6 \mathrm{~km} \mathrm{SSMF}$ using a $20-\mathrm{GHz}$ VCSEL at 1525 nm," J. Light. Technol., vol. 35, no. 8, pp. 1342-1349, Feb. 2017.

[73] A. Dochhan, N. Eiselt, R. Hohenleitner, H. Griesser, M. Eiselt, M Ortsiefer, C. Neumeyr, J. J. V. Olmos, I. T. Monroy, and J.-P. Elbers, "56 $\mathrm{Gb} / \mathrm{s}$ DMT transmission with VCSELs in 1.5 um wavelength range over up to $12 \mathrm{~km}$ for DWDM intra-data center connects," in Proc. ECOC, Düsseldorf, Sep. 2016.

[74] K. Zhong, X. Zhou, J. Huo, C. Yu, C. Lu, and A. P. T. Lau, "digital signal processing for short-reach optical communications: a review of current technologies and future trends," J. Lightw. Technol., vol. 36, no. 2, pp. 377-400, Jan. 2018.

[75] R. Lin, J. Van Kerrebrouck, X. Pang, M. Verplaetse, O. Ozolins, A. Udalcovs, L. Zhang, L. Gan, M. Tang, S. Fu, R. Schatz, U. Westergren, S. Popov, D. Liu, W. Tong, T. De Keulenaer, G. Torfs, J. Bauwelinck, X. Yin, and J. Chen, "Spatial division multiplexing for optical data center networks," in Proc. ONDM, Dublin, May 2018.

[76] R. Lin, X. Pang, J. Van Kerrebrouck, M. Verplaetse, O. Ozolins, A. Udalcovs, L. Zhang, L. Gan, M. Tang, S. Fu, R. Schatz, U. Westergren, S. Popov, D. Liu, W. Tong, T. De Keulenaer, G. Torfs, J. Bauwelinck, X. Yin, and J. Chen, "Real-time $100 \mathrm{Gbps} / \lambda /$ core NRZ and EDB IM/DD transmission over $10 \mathrm{~km}$ multicore fiber," in Proc. OFC, San Diego, Mar. 2018.

[77] R. Lin, J. Van Kerrebrouck, X. Pang, M. Verplaetse, O. Ozolins, A. Udalcovs, L. Zhang, L. Gan, M. Tang, S. Fu, R. Schatz, U. Westergren, S. Popov, D. Liu, W. Tong, T. De Keulenaer, G. Torfs, J. Bauwelinck, X. Yin, and J. Chen, "Real-time $100 \mathrm{Gbps} / \lambda /$ core NRZ and EDB IM/DD transmission over multicore fiber for intra-datacenter communication networks," Opt. Exp., vol. 26, no. 8, pp. 10519-10526, 2018.

[78] O. Ozolins, X. Pang, A. Udalcovs, R. Lin, J. Van Kerrebrouck, L. Gan, L. Zhang, M. Tang, S. Fu, R. Schatz, U. Westergren, G. Jacobsen, D. Liu, W. Tong, G. Torfs, J. Bauwelinck, J. Chen, S. Popov, and X. Yin, " $7 \times 149$ Gbit/s PAM4 transmission over $1 \mathrm{~km}$ multicore fiber for short-reach optical interconnects," in Proc. CLEO: Science and Innovations, May 2018.

[79] X. Pang, J. Van Kerrebrouck, O. Ozolins, R. Lin, A. Udalcovs, L. Zhang, S. Spiga, M. C Amann, G. Van Steenberge, L. Gan, M. Tang, S. Fu, R. J Schatz, G. Jacobsen, S. Popov, D. Liu, G. Torfs, J. Bauwelinck, X. Yin, and J. Chen, "7× 100 Gbps PAM-4 transmission over 1-km and 10-km single mode 7-core fiber using 1.5- $\mu \mathrm{m}$ SM-VCSEL," in Proc. OFC, San Diego, Mar. 2018

[80] X. Pang, J. V. Kerrebrouck, O. Ozolins, R. Lin, A. Udalcovs, L. Zhang, S. Spiga, M. C. Amann, G. V. Steenberge, L. Gan, M. Tang, S. Fu, R. Schatz, G. Jacobsen, S. Popov, D. Liu, W. Tong, G. Torfs, J. Bauwelinck, X. Yin, and J. Chen, "High-speed SDM interconnects with directly-modulated 1.5- $\mu \mathrm{m}$ VCSEL enabled by low-complexity signal processing techniques," in Proc. SPPCOM, San Diego, Mar. 2018.

[81] J. Van Kerrebrouck, X. Pang, O. Ozolins, R. Lin, A. Udalcovs, L. Zhang, H. Li, S. Spiga, M. C. Amann, L. Gan, M. Tang, S. Fu, R. Schatz, G. Jacobsen, S. Popov, D. Liu, W. Tong, G. Torfs, J. Bauwelinck, J. Chen, and X. Yin, "High-speed PAM4-based optical SDM interconnects with directly modulated long-wavelength VCSEL," J. Lightw. Technol., vol. 37, no. 2, pp. 356-362, Jan. 2019.

[82] J. V. Kerrebrouck, L. Zhang, R. Lin, X. Pang, A. Udalcovs, O. Ozolins, S. Spiga, MC Amann, G. Van Steenberge, L. Gan, M. Tang, S. Fu, R. Schatz, S. Popov, D. Liu, W. Tong, S. Xiao, G. Torfs, J. Chen, J. Bauwelinck, and $\mathrm{X}$. Yin, "726.7-Gb/s 1.5- $\mu \mathrm{m}$ single-mode VCSEL discrete multi-tone transmission over 2.5-km multicore fiber," in Proc. OFC, San Diego, Mar. 2018.

[83] E. Ip, M. J. Huang, K. Bennett, Y. K. Huang, A. Tanaka, A. Korolev, K. Koreshkov, W. Wood, E. Mateo, J. Hu, and Y. Yano, " $146 \lambda \times 6 \times 19-$ Gbaud wavelength-and mode-division multiplexed transmission over $10 \times 50-\mathrm{km}$ spans of few-mode fiber with a gain-equalized few-mode EDFA," in Proc. OFC, Anaheim, Mar. 2013.

[84] K. Igarashi, D. Souma, Y. Wakayama, K. Takeshima, Y. Kawaguchi, T. Tsuritani, I. Morita, and M. Suzuki, "114 space-division-multiplexed transmission over 9.8-km weakly-coupled-6-mode uncoupled-19-core fibers," in Proc. OFC, Los Angeles, Mar. 2015.

[85] M.S. Faruk, and K. Kikuchi, "Adaptive frequency-domain equalization in digital coherent optical receivers," Opt. Exp., vol. 19, no. 13, pp. 12789-12798, Jun. 2011

[86] N. Bai, and G. Li, "Adaptive frequency-domain equalization for mode division multiplexed transmission," IEEE Photon. Technol. Lett., vol. 24, no. 21, pp. 1918-1921, Sep. 2012.

[87] R. Ryf, R. J. Essiambre, S. Randel, A. H. Gnauck, P. J. Winzer, T. Hayashi, T. Taru and T. Sasaki, "MIMO-Based Crosstalk Suppression in Spatially Multiplexed 3 56-Gb/s PDM-QPSK Signals for Strongly Coupled Three-Core Fiber," Photon. Technol. Lett., vol. 23, no. 20, pp. 1469-1471, Oct. 2011.

[88] F. Karinou, C. Prodaniuc, N. Stojanovic, M. Ortsiefer, A. Daly, R. Hohenleitner, B. Kögel, and C. Neumeyr, "Directly PAM-4 modulated 1530-nm VCSEL enabling $56 \mathrm{~Gb} / \mathrm{s} / \lambda$ data-center interconnects," Photon. Technol. Lett., vol. 27, no. 17, pp. 1872-1875, 2015.

[89] G. Chen, J. Du, L. Sun, W. Zhang, K. Xu, X. Chen, G. T. Reed, and Z. He, "Nonlinear distortion mitigation by machine learning of SVM classification for PAM-4 and PAM-8 modulated optical interconnection," J. Lightw. Technol., vol. 36, no. 3, pp. 650-657, 2018.

[90] L. Zhang, O. Ozolins, R. Lin, A. Udalcovs, X. Pang, L. Gan, R. Schatz, A. Djupsjöbacka, J. Mårtensson, U. Westergren, M. Tang, S. Fu, D. Liu, W. Tong, S. Popov, G. Jacobsen, W. Hu, S. Xiao and J. Chen, "Kernel adaptive filtering for nonlinearity-tolerant optical direct detection systems," in Proc. ECOC, Rome, Sep. 2018.

[91] L. Zhang, J. Van Kerrebrouck, O. Ozolins, R. Lin, X. Pang, A. Udalcovs, S. Spiga, MC Amann, G. Van Steenberge, L. Gan, M. Tang, S. Fu, R. Schatz, S. Popov, D. Liu, W. Tong, G. Torfs, J. Bauwelinck, X. Yin, S. Xiao and J. Chen, "Experimental demonstration of 503.61-Gbit/s DMT over 10-km 7-core fiber with 1.5- $\mu \mathrm{m}$ SM-VCSEL for optical interconnects," in Proc. ECOC, Rome, Sep. 2018.

[92] A. Udalcovs, X. Pang, O. Ozolins, R. Lin, L. Gan, R. Schatz, A Djupsjöbacka, J. Mårtensson, M. Tang, S. Fu, D. Liu, W. Tong, J. Chen, S. Popov, and G. Jacobsen, "MCF-Enabled Self-Homodyne 16/64QAM Transmission for SDM Optical Access Networks," in Proc. CLEO, San Jose, May, 2018.

[93] D. Klonidis, F. Cugini, O. Gerstel, M. Jinno, V. Lopez, E. Palkopoulou, M. Sekiya, D. Siracusa, G. Thouenon, and C. Betoule, "Spectrally and spatially flexible optical network planning and operations," IEEE Commun. Mag., vol. 53, no. 2, pp. 69-78, Feb. 2015.

[94] A. Muhammad, G. Zervas, D. Simeonidou, and R. Forchheimer, "Routing, spectrum and core allocation in flexgrid SDM networks with multi-core fibers," in Proc. ONDM, Stockholm, May 2014.

[95] P. S. Khodashenas, J. Moscoso, D. Siracusa, F. Pederzolli, B. Shariati, D. Klonidis, E. Salvadori, and I. Tomkos, "Comparison of spectral and spatial super-channel allocation schemes for SDM networks," J. Lightwave Technol., vol. 34, no. 11, pp. 2710-2716, Jun. 2016.

[96] B. Shariati, A. Mastropaolo, N. P. Diamantopoulos, J. M. Rivas-Moscoso, F. Pederzolli, D. Siracusa, D. Klonidis, and I. Tomkos, "Spectrally-Spatially Flexible Optical Networking," in Proc. ACP, paper AS2D.1, Wuhan China, Nov. 2016.

[97] M. Fiorani, M. Tornatore, J. Chen, L. Wosinska, and B. Mukherjee, "Spatial division multiplexing for high capacity optical interconnects in modular data centers," J. Opt. Commun. Netw., vol. 9, no. 2, pp. A143-A153, Feb. 2017.

[98] D. Marom, and M. Blau, "Switching solutions for WDM-SDM optical networks," IEEE Commun. Mag., vol. 53, no. 2, pp. 60-68, Feb. 2015.

[99] R. Ryf, S. Chandrasekhar, S. Randel, D. T. Neilson, N. K. Fontaine, and M. Feuer, "Physical layer transmission and switching solutions in support of spectrally and spatially flexible optical networks," IEEE Commun. Mag., vol. 53, no. 2, pp. 52-59, Feb. 2015.

[100] H. Yuan, M. Furdek, A. Muhammad, A. Saljoghei, L. Wosinska, and G. Zervas, "Space-division multiplexing in data solutions and crosstalk-suppressed resource allocation," J. Opt. Commun. Netw., vol. 10, no. 4, pp. 272-288, Apr. 2018

[101] Z. Zhu, and S. Zhong, "OvS: A distributed and WDM/SDM enabled optical switching fabric for intra-data center networking," in Proc. APC, paper PW3B.2, San Diego, Jul. 2014.

[102] Z. Zhu, S. Zhong, L. Chen, and K. Chen, "Fully programmable and scalable optical switching fabric for petabyte data center," Opt. Exp., vol. 23, no. 3, pp. 3563-3580, Feb. 2015

[103] Core and Pod Data Center Design - Big Switch Networks, http://go.bigswitch.com/rs/974-WXR-561/images/Core-and-Pod\%20Over 
view.pdf

[104] S. Fujii, Y. Hirota, H. Tode, and K. Murakami, "On-demand spectrum and core allocation for reducing crosstalk in multicore fibers in elastic optical networks," J. Opt. Commun. Netw., vol. 6, no. 12, pp. 1059-1071, Sep. 2014.

[105] M. Y. Namaad, A. G. Rahbar, and B. Alizadeh, "Adaptive modulation and flexible resource allocation in space-division-multiplexed elastic optical networks," J. Opt. Commun. Netw., vol. 10, no. 3, pp. 240-251, Mar. 2018.

[106] M. N. Dharmaweera, L. Yan, M. Karlsson, and E. Agrell, "Nonlinear-impairments- and crosstalk-aware resource allocation schemes for multicore-fiber-based flexgrid networks," in Proc. ECOC, Düsseldorf, Sep. 2016.

[107] M. Yang, Y. Zhang, and Q. Wu, "Routing, spectrum, and core assignment in SDM-EONs with MCF: node-arc ILP/MILP methods and an efficient XT-aware heuristic algorithm," J. Opt. Commun. Netw., vol. 10, no. 3, pp. 195-208, Mar. 2018.

[108] P. S. Khodashenas, J. M. Rivas-Moscoso, D. Siracusa, F. Pederzolli, B. Shariati, D. Klonidis, E. Salvadori, and I. Tomkos, "Comparison of spectral and spatial super-channel allocation schemes for SDM networks," J. Lightwave Technol., vol. 34, no. 11, pp. 2710-2716, Jun. 2016.

[109] H. Tode, and Y. Hirota, "Routing, spectrum and core assignment for space division multiplexing elastic optical networks," in Proc. 16th International Telecommunications Network Strategy and Planning Symposium (Networks), Funchal, 2014.

[110] L. Yan, M. Fiorani, A. Muhammad, M. Tornatore, E. Agrell, and L. Wosinska, "Network performance trade-off in modular data centers with optical spatial division multiplexing," J. Opt. Commun. Netw., vol. 10, no. 9, pp. 796-808, Sept. 2018.

[111] Peter X. Gao, A. Narayan, S. Karandikar, J. Carreira, and S. Han, R. Agarwal, S. Ratnasamy, and S. Shenker, "Network requirements for resource disaggregation," in Proc. OSDI, Savannah, pp. 249-264, Nov. 2016.

[112] A. Saljoghei, H. Yuan, V. Mishra, M. Enrico, N. Parsons, C. Kochis, P. D. Dobbelaere, D. Theodoropoulos, D. Pnevmatikatos, D. Syrivelis, A. Reale, T. Hayashi, T. Nakanishi, and G. Zervas, "MCF-SMF hybrid low-latency circuit switched optical network for disaggregated data centers," J. Lightw. Technol., vol. 37, no. 16, pp. 4017 - 4029, Aug. 2019. 